J. DIFFERENTIAL GEOMETRY

70 (2005) 453-472

\title{
LOWER BOUNDS ON THE CALABI FUNCTIONAL
}

\author{
S.K. DONALDSON \\ Dedicated to Raoul Bott
}

\section{Introduction}

A cornerstone of Atiyah and Bott's treatment [1] of Yang-Mills theory over Riemann surfaces is a relation they discovered between the YangMills functional and filtrations of a holomorphic bundle. The relation can be stated as follows. Let $\Sigma$ be a compact Riemann surface with a fixed compatible metric, normalised to have area $4 \pi^{2}$. Let $E$ be a holomorphic vector bundle over $\Sigma$ and consider a flag $\mathcal{F}$ of sub-bundles

$$
0=E_{0} \subset E_{1} \cdots \subset E_{q}=E .
$$

Recall that for any bundle $V$ over $\Sigma$, the slope $\mu(V)$ of $V$ is defined to be the quotient of the degree of $\mathrm{V}$ by the rank. We say the flag $\mathcal{F}$ is slope-decreasing if $\mu\left(E_{1}\right)>\mu\left(E_{2}\right) \cdots>\mu(E)$. Equivalently, the quotients $Q_{i}=E_{i} / E_{i-1}$ have $\mu\left(Q_{1}\right)>\cdots>\mu\left(Q_{q}\right)$. Define

$$
\Phi(\mathcal{F})=\left(\sum_{i=1}^{q} \mu\left(Q_{i}\right)^{2} \operatorname{rank}\left(Q_{i}\right)\right)^{1 / 2} .
$$

Atiyah and Bott relate this algebro-geometric quantity to the YangMills functional - the $L^{2}$ norm of the curvature $F_{A}$-restricted to compatible unitary connections $A$ on $E$. Their result is

$$
\inf _{A}\left\|F_{A}\right\|=\sup _{\mathcal{F}, \operatorname{dec}} \Phi(\mathcal{F})
$$

where on the right-hand side the supremum runs over the slope-decreasing flags. In fact, this supremum is attained by the canonical Harder-Narasimhan filtration of $E$. The infimum on the left-hand side is not in general attained: this happens if and only if $E$ is a direct sum of stable bundles. Notice that there is an easy lower bound $\left\|F_{A}\right\| \geq \mu(E) \sqrt{\operatorname{rank}(E)}$ deriving from the fact that $\frac{i}{2 \pi} \operatorname{Tr}\left(F_{A}\right)$ represents $c_{1}(E)$. This trivial lower bound is just $\Phi\left(\mathcal{F}^{0}\right)$ where $\mathcal{F}^{0}$ is the trivial flag (with $q=1$ ). So $\sup _{\mathcal{F}, \operatorname{dec}} \Phi(\mathcal{F}) \geq \mu(E) \sqrt{\operatorname{rank} E}$, and it is easy to see that equality holds if and only if the bundle $E$ is semi-stable, i.e., if there is no non-trivial slope-decreasing flag.

The Atiyah-Bott result can be viewed as two statements: $\inf _{A} \geq \sup _{\mathcal{F}}$ and $\inf _{A} \leq \sup _{\mathcal{F}}$. The proof of the first of these involves a simple

Received 06/24/2005. 
differential-geometric argument, turning on the principle that "curvature decreases in holomorphic sub-bundles and increases in holomorphic quotients" ([1] , Proposition 8.13 and the remark in the second paragraph of p. 575). The proof of the second relies on the theorem of Narasamihan and Seshadri on the existence of projectively flat unitary connections on stable bundles.

In [3], Calabi began the study of the $L^{2}$ norm of the scalar curvature of Kahler metrics, running over a fixed Kahler class on a compact Kahler manifold. This functional is equivalent to the $L^{2}$-norm of the full curvature tensor, in that the two differ by topological terms. The purpose of this paper is to establish an analogue of (one half of) the Atiyah-Bott result for this Calabi functional (and some variants for $L^{p}$ norms). Our result bears on the algebraic case, so we suppose that $X$ is a smooth complex projective variety, that $L \rightarrow X$ is a fixed ample line bundle and we consider Kahler metrics $\omega$ in the class $c_{1}(L)$. We use the notion, introduced in [8], of a test configuration $\mathcal{X}$ for $X$. The detailed definition will be reviewed below, but in essence this comprises a $\mathbf{C}^{*}$-equivariant family $\pi: \mathcal{X} \rightarrow \mathbf{C}$ with generic fibre $X_{t}=\pi^{-1}(t)$ isomorphic to $X$, for $t \neq 0$. The central fibre $X_{0}$ need not be isomorphic to $X$, it may be a highly singular variety or even a scheme, but it has a $\mathbf{C}^{*}$-action and the definition requires a lift of this action to a line bundle $\mathcal{L}$. We will define a numerical invariant $\Psi(\mathcal{X})$ using the $\mathbf{C}^{*}$ action on the vector spaces $H^{0}\left(X_{0}, \mathcal{L}^{k}\right)$, related to the generalised Futaki invariant. In stating our results, it is convenient to work with a quantity $S(\omega)$ defined to be $\frac{1}{4 \pi}$ times the usual scalar curvature of the metric $\omega$. (Thus, $S(\omega) \frac{\omega^{n}}{n !}=\frac{1}{2} \rho \wedge \frac{\omega^{n-1}}{(n-1) !}$, where $\rho$ is the Ricci form representing $c_{1}(X)$.) Then, our result takes the form

$$
\inf _{\omega}\|S(\omega)\|_{L^{2}} \geq \sup _{\mathcal{X}} \Psi(\mathcal{X}) .
$$

There is a rather trivial lower bound on the Calabi functional, deriving from the fact that the integral of $S(\omega)$ yields $\left\langle\frac{1}{2(n-1) !} c_{1}(X) \cup\right.$ $\left.\omega^{n-1},[X]\right\rangle$, so the average value $\hat{S}$ is a topological invariant of the Kahler class. Thus

$$
\|S(\omega)\|_{L^{2}}^{2}=\|S-\hat{S}\|_{L^{2}}^{2}+\hat{S}^{2} \operatorname{Vol}(X) \geq \hat{S}^{2} \operatorname{Vol}(X) .
$$

The essential feature of our definition of $\Psi(\mathcal{X})$ is that we get a better bound than this precisely when $X$ is not "K-semistable" in the sense of [8], just as in the Atiyah-Bott case. (There is also an extension of this discussion to extremal metrics, see Section 2 below.) Thus, one immediate consequence of our Theorem (as Richard Thomas pointed out to the author) is a new and simpler proof of the fact that the existence of a constant scalar curvature Kahler metric implies K-semistability. This 
argument does not need any special constructions (as in [14]), in the case when the manifold has holomorphic vector fields.

It is natural to conjecture that in fact

$$
\inf _{\omega}\|S(\omega)\|_{L^{2}}=\sup _{\mathcal{X}} \Psi(\mathcal{X})
$$

This would be a variant of the conjectures in $[\mathbf{2 3}],[\mathbf{2 1}],[\mathbf{8}]$ relating K-stability to the existence of constant scalar curvature metrics (and the extension of that discussion to extremal metrics in [19]). However, neither conjecture immediately implies the other.

We will explain the analogy with the Atiyah-Bott result in somewhat more detail in Section 2. Both set-ups fit, formally, into the more general framework of "moment maps and stability" discussed in [7] for example. The results can be seen as infinite-dimensional versions of part of the theory developed by Kirwan $[\mathbf{1 2}]$ in finite dimensions. The method of proof, we use here is that of finite-dimensional approximation, in the mould of $[\mathbf{9}],[\mathbf{1 0}]$. That is, we derive our inequalities by studying the asymptotics of finite-dimensional problems, essentially of the kind considered by Kirwan. However, the proof of the result in this paper is substantially simpler than that in $[\mathbf{9}]$. In particular, we do not need to make use of the notion of "balanced" metrics. The essential ingredient in our proof is the asymptotic expansion for the "density of states" function due to Yau, Tian, Zelditch, Liu, Catlin and Ruan [20], [24], [13], [4], [18]. We also need some discussion of singular varieties and schemes to allow us to apply the general moment map theory. The author first saw that this could be done using the point of view developed in the work of Zhang [25], Phong and Sturm [15], [16] involving the "Chow norm" and an action on Chow vectors. However, it turns out that one can avoid appealing to these concepts explicitly and we only need some comparatively straightforward technical facts to handle these issues of singularities (notably a result related to the equivariant Riemann-Roch Theorem, Proposition 3 below).

In 1997, and intermittently since, the author has discussed with X-X. Chen the possibility of obtaining lower bounds on the Calabi functional using a similar pattern of argument, but replacing the finite-dimensional approximations by the use of "geodesic rays" in the space of Kahler potentials, in the manner of $[\mathbf{5}],[\mathbf{6}]$. This would have independent interest and Chen and the author hope to discuss this work in a future article.

The author is grateful to Richard Thomas and Xiu-Xiong Chen for discussions of these topics. 
S.K. DONALDSON

\section{Test configurations}

We begin by recalling the definition of a test configuration from [7]. Given an ample line bundle $L \rightarrow X$, a test configuration for the pair $(X, L)$ consists of:

- a scheme $\mathcal{X}$ with a $\mathbf{C}^{*}$ action;

- a flat $\mathbf{C}^{*}$-equivariant map $\pi: \mathcal{X} \rightarrow \mathbf{C}$, with fibres $X_{t}$;

- an equivariant line bundle $\mathcal{L} \rightarrow \mathcal{X}$, ample on all fibres;

- for some $r>0$, an isomorphism of the pair $\left(X_{1},\left.\mathcal{L}\right|_{X_{1}}\right)$ with the original pair $\left(X, L^{r}\right)$.

Thus, we have a sequence of vector spaces $U_{k}=H^{0}\left(X_{0},\left.\mathcal{L}\right|_{X_{0}} ^{k}\right)$ with $\mathbf{C}^{*}$-actions. Let $A_{k}: U_{k} \rightarrow U_{k}$ be the endomorphisms generating these actions (so $e^{t} \in \mathbf{C}^{*}$ acts as $e^{t A_{k}}$ on $U_{k}$ ). We are interested in the asymptotic behaviour, with respect to $k$, of the dimension of $U_{k}$ and the total weight of the action, i.e., the trace of the $A_{k}$. These are given by Hilbert polynomials:

$$
\begin{gathered}
\operatorname{dim} U_{k}=a_{0} k^{n}+a_{1} k^{n-1}+\cdots, \\
\operatorname{Tr} A_{k}=b_{0} k^{n+1}+b_{1} k^{n}+\cdots,
\end{gathered}
$$

for large $k$, as discussed in [7], [17]. We go one step further and define a positive number $Q$ by the leading term in the polynomial defining the trace of the squares:

$$
\operatorname{Tr} A_{k}^{2} \sim Q k^{n+2} .
$$

It is clear that $Q \geq 0$, one way of seeing that $Q$ is strictly positive is to use (20) in Section 5 below. Notice that, from general theory, the $a_{i}, b_{i}$ and $Q$ are rational numbers.

Now, we define

$$
\Psi(\mathcal{X})=-\frac{1}{r^{(n-2) / 2}} \frac{b_{1}}{\sqrt{Q}} .
$$

The normalisation by $1 / r^{(n-2) / 2}$ means that we do not change $\Psi(\mathcal{X})$ if we replace $\mathcal{L}$ by a positive power $\mathcal{L}^{s}$. Also this scaling weight is the same as that of the Calabi functional

$$
r^{(n-2) / 2}\|S(r \omega)\|_{L^{2}(r \omega)}=\|S(\omega)\|_{L^{2}(\omega)} .
$$

The upshot of this is that in proving our theorem, we can always suppose $r=1$, which we do from now on. By the flatness of the family and the Riemann-Roch Theorem for $X$, we can identify the co-efficient $a_{0}$ with the volume of $X$ and $a_{1}$ with the integral of $S(\omega)$ for any metric $\omega$ representing $c_{1}(L)$. Thus, $\hat{S}=a_{1} / a_{0}$ and the trivial lower bound (4) is $\|S\| \geq\left|a_{1}\right| / \sqrt{a_{0}}$.

There are two ways in which we can modify a test configuration. First, we can pull back $\mathcal{X}$ by a $d$-fold covering of the base. This changes 
$A_{k}$ to $d A_{k}$ and plainly does not affect $\Psi$. Second, we can change the $\mathbf{C}^{*}$ action on the line bundle $\mathcal{L}$ by a character $\lambda \mapsto \lambda^{\nu}$ of $\mathbf{C}^{*}$. This changes $A_{k}$ to $A_{k}+k \nu 1$ and so $b_{i}$ to $\tilde{b}_{i}=b_{i}+\nu a_{i}$, and $Q$ to $\tilde{Q}=Q+2 b_{0} \nu+a_{0} \nu^{2}$. Thus, for the new configuration $\tilde{\mathcal{X}}$, we have

$$
\Psi(\tilde{\mathcal{X}})=-\frac{b_{1}+\nu a_{1}}{\sqrt{Q+2 b_{0} \nu+a_{0} \nu^{2}}} .
$$

We consider the supremum of this expression over $\nu$, initially regarded as a real variable. The analysis of this supremum brings in the Futaki invariant $F_{\mathcal{X}}$ of the test configuration. This is defined to be

$$
F_{\mathcal{X}}=b_{1}-\frac{b_{0} a_{1}}{a_{0}}
$$

(This terminology differs by a factor of $a_{0}$ from that in [8], where only the sign of $F_{\mathcal{X}}$ was relevant.) Then, as the reader will easily verify, if $F \mathcal{X}<0$ the expression in (9) above is maximised when

$$
\nu=\frac{a_{1} Q-b_{0} b_{1}}{a_{0} b_{1}-a_{1} b_{0}} .
$$

By taking a covering we may suppose that this is an integer, so the supremum is realised by some test configuration. On the other hand, if $F_{\mathcal{X}} \geq 0$, the supremum is not attained for finite $\nu$ but occurs in the limit as $\nu$ tends to $\infty$ or $-\infty$, depending on the sign of $a_{1}$. In this second case, the supremum is just $\left|a_{1}\right| / \sqrt{a_{0}}$ which is just the trivial lower bound on $\|S\|$. In the first case, when the supremum is attained, a little calculation shows that the supremum is

$$
\left(\frac{\left|a_{1}\right|^{2}}{a_{0}}+\hat{\Psi}_{\mathcal{X}}^{2}\right)^{1 / 2}
$$

where we define

$$
\hat{\Psi}_{\mathcal{X}}=-\frac{F_{\mathcal{X}}}{\sqrt{Q-b_{1}^{2} / a_{0}}} .
$$

The denominator here has a natural interpretation. We write $\underline{A}_{k}: U_{k} \rightarrow$ $U_{k}$ for the trace-free part of $A_{k}$, i.e.,

$$
\underline{A}_{k}=A_{k}-\frac{\operatorname{Tr} A_{k}}{\operatorname{dim} U_{k}} 1 \text {. }
$$

Then as $k \rightarrow \infty$,

$$
\operatorname{Tr} \underline{A}_{k}^{2} \sim\left(Q-b_{1}^{2} / a_{0}\right) k^{n+2}
$$

With this discussion in place, we can state our main theorem precisely.

Theorem 1. If $\mathcal{X}$ is a test configuration for the pair $(X, L)$, then for any Kahler metric $\omega$ in the class $c_{1}(L)$, we have

$$
\|S(\omega)\|_{L^{2}} \geq \Psi(\mathcal{X}) .
$$


By the discussion above, it is completely equivalent to prove that for any test configuration

$$
\|S(\omega)-\hat{S}\|_{L^{2}} \geq-\frac{F_{\mathcal{X}}}{Q-\frac{b_{1}^{2}}{a_{0}}},
$$

and it is in this form that we shall prove the result. Notice that the inequality is vacuous unless $F_{\mathcal{X}}<0$, that is, unless $(X, L)$ is not $\mathrm{K}$ semistable. We will prove a more general result dealing with $L^{q}$ norms (loosely analogous to the discussion of other norms in $[\mathbf{1}]$ ). For an even integer $p$, we define $N_{p}(\mathcal{X})>0$ by the leading term

$$
\operatorname{Tr} \underline{A}_{k}^{p} \sim N_{p}^{p} k^{n+p}
$$

in the appropriate Hilbert polynomial. Thus, when $p=2$,

$$
Q-\frac{b_{1}^{2}}{a_{0}}=N_{2}^{2} .
$$

Momentarily re-instating the integer $r$ in the definition of a test configuration, we define

$$
\hat{\Psi}_{p}(\mathcal{X})=-\frac{1}{r^{(n / q)-1}} \frac{F_{\mathcal{X}}}{N_{p}(\mathcal{X})} .
$$

Again, the power of $r$ is chosen so that this is unchanged if we replace $\mathcal{L}$ by $\mathcal{L}^{s}$.

Theorem 2. If $\mathcal{X}$ is any test configuration for $(X, L)$, then for any metric $\omega$ in the class $c_{1}(L)$, we have

$$
\|S(\omega)-\hat{S}\|_{L^{q}} \geq \hat{\Psi}_{p}(\mathcal{X}),
$$

where $p$ is any even integer and $q$ is the conjugate exponent to $p$ (i.e., $\left.p^{-1}+q^{-1}=1\right)$.

Just as before, one can check that that the scaling weight of the $L^{q}$ norm of $S(\omega)$ agrees with the power of $r$ in (11), so we can reduce to the case when $r=1$. By the preceding discussion, Theorem 2 (in the case when $p=2$ ) implies Theorem 1 , and in the body of the paper below we prove Theorem 2. (We remark that, when $p \neq 2$, there is no simple exact relation between the $L^{q}$ norms of $S$ and $S-\hat{S}$. One can derive slightly different lower bounds for the $L^{q}$ norms of $S$ using the techniques of this paper, but we find it simpler to work with $S-\hat{S}$.)

If the automomorphism group of the pair $(X, L)$ contains a non-trivial compact connected subgroup $G$, then there is another relatively elementary lower bound on the Calabi functional, obtained from the Futaki invariant in its original, differential geometric, form. We consider a $G$ invariant metric on $X$. Then, we get a Lie algebra homomorphism from 
$\operatorname{Lie}(G)$ to the functions on $X$, under Poisson bracket. Let $\xi$ be an element of $\operatorname{Lie}(G)$ and let $H$ be the corresponding Hamiltonian. Then, the integrals

$$
\int_{X} S H d \mu \quad \int_{X} H^{p} d \mu
$$

are topological invariants of the data: they do not change as we vary $\omega$ among $G$-invariant metrics in the same Kahler class. By Holder's inequality, we have

$$
\|S-\hat{S}\|_{L^{q}} \geq \frac{1}{\|H-\hat{H}\|_{L^{p}}} \int_{X}(S-\hat{S}) H d \mu
$$

where $\hat{H}$ is the average value of $H$, and the right-hand side is a topological invariant of the data.

We will now explain how to derive this as a special case of our Theorem 2. By a density argument, we can suppose that $\xi$ generates a circle action on $(X, L)$ which we extend to a holomorphic $\mathbf{C}^{*}$ action. Then, we define a test configuration by taking the product $\mathcal{X}=X \times \mathbf{C}$ but using this non-trivial $\mathbf{C}^{*}$ action. Thus, in this case, the central fibre $\left(X_{0}, \mathcal{L}\right)$ is just the original pair $(X, L)$, with the given $\mathbf{C}^{*}$ action. The key point now is that

$$
b_{0}=\int_{X} H d \mu, b_{1}=\int_{X} S H d \mu
$$

so that

$$
F_{\mathcal{X}}=\int_{X}(S-\hat{S}) H d \mu
$$

Thus, the algebro-geometric definition of the Futaki invariant reduces to the differential geometric one. This is explained in Prop. 2.2.2 of [8] and we will obtain a generalisation of the fact in Proposition 3 below. Moreover, the same discussion shows that, for even integers $p$,

$$
N_{p}=\|H-\hat{H}\|_{L^{p}} .
$$

Changing the sign of $\xi$, if necessary, we can suppose that the Futaki invariant is negative. Then, (12) follows as a special case of our Theorem 2. In fact, our Theorem gives rather more even in this case, since the lower bound is obtained for arbitrary metrics in the Kahler class, not just the $G$-invariant ones.

We return now to a situation considered by Atiyah and Bott and explain how their result can be cast in a similar form as ours. Let $\mathcal{F}$ be a flag of sub-bundles in $E \rightarrow \Sigma$, as before. Let $W=\left(w_{1}, \ldots, w_{q}\right)$ be a vector of strictly increasing integers. Then, one can construct a degeneration $\mathcal{E}=\mathcal{E}(\mathcal{F}, W)$ from this data. This is a $\mathbf{C}^{*}$-equivariant bundle over $\mathbf{C}^{*} \times \Sigma$ which is isomorphic to $E$ on each slice $\{t\} \times \Sigma$ for $t \neq 0$ and to $E_{0}=Q_{1} \oplus Q_{2} \cdots \oplus Q_{q}$ when $t=0$, with the property that the $\mathbf{C}^{*}$-action on $E_{0}$ has weight $w_{i}$ on the summand $Q_{i}$. We fix a square 
root $K_{\Sigma}^{1 / 2}$ of the canonical bundle and any line bundle $L \rightarrow \Sigma$ of degree 1. Now, we consider the $\mathbf{C}^{*}$ action on the vector spaces

$$
U_{k}=H^{0}\left(E_{0} \otimes K_{\Sigma}^{1 / 2} \otimes L^{k}\right),
$$

with generators $A_{k}: U_{k} \rightarrow U_{k}$. Following just the same pattern as before, we look at the large $k$ behaviour,

$$
\begin{gathered}
\operatorname{dim} U_{k}=\alpha_{0} k+\alpha_{1}, \\
\operatorname{Tr} A_{k}=\beta_{0} k+\beta_{1}, \\
\operatorname{Tr} A_{k}^{2} \sim Q k,
\end{gathered}
$$

and we define

$$
\Psi(\mathcal{F}, W)=-\frac{\beta_{1}}{\sqrt{Q}} .
$$

Lemma 1. For any bundle $E \rightarrow \Sigma$ we have

$$
\sup _{\mathcal{F}, W} \Psi(\mathcal{F}, W)=\sup _{\mathcal{F}, \operatorname{dec}} \Phi(\mathcal{F}),
$$

where on the left-hand side, the supremum is taken over arbitrary flags $\mathcal{F}$ and increasing weight vectors $W$ and on the right-hand side the supremum is taken over slope-decreasing flags $\mathcal{F}$.

Given a flag $\mathcal{F}$ and weight vector $W$, we have

$$
U_{k}=\bigoplus H^{0}\left(Q_{i} \otimes K_{\Sigma}^{1 / 2} \otimes L^{k}\right),
$$

with the action of weight $w_{i}$ on the the ith summand. It follows that

$$
\alpha_{0}=\sum r_{i}, \alpha_{1}=\sum d_{i}, \beta_{0}=\sum r_{i} w_{i}, \beta_{1}=\sum d_{i} w_{i}, Q=\sum r_{i} w_{i}^{2},
$$

where $Q_{i}$ has rank $r_{i}$ and degree $d_{i}$. So, we obtain

$$
\Psi(\mathcal{F}, W)=-\frac{\sum d_{i} w_{i}}{\sqrt{\sum r_{i} w_{i}^{2}}} .
$$

Now, suppose that $\mathcal{F}$ is slope-decreasing. We maximise $\Psi(\mathcal{F}, W)$ over weights $w_{i}$, initially regarded as arbitrary real numbers. The maximum occurs at $w_{i}^{\max }=-C d_{i} / r_{i}$ for any constant $C$. Taking a suitable $C$, we can suppose that $w_{i}^{\max }$ are integers, but more crucially, the condition that $\mathcal{F}$ was a slope-decreasing flag means that the $w_{i}^{\max }$ are increasing. Then, we have

$$
\Psi\left(\mathcal{F}, W^{\max }\right)=\Phi(\mathcal{F}),
$$

so we have established one half of the Proposition, i.e., $\sup \Psi \geq \sup \Phi$. We prove the other half by induction on the length $q$ of the flag. We take as inductive proposition the statement that for any flag $\mathcal{F}$ of length $q$ and any weakly increasing weight vector $W$ (i.e., with $w_{1} \leq \cdots \leq w_{q}$ ) we have $\Psi(\mathcal{F}, W) \leq \sup _{\mathcal{F} \text {,dec }} \Phi$, where $\Psi(\mathcal{F}, W)$ is defined by (13). This is clearly true for $q=1$. Suppose that $\mathcal{F}$ is any flag and $W^{0}$ is a weaklyincreasing weight vector. We maximise the expression for $\Psi(\mathcal{F}, W)$ over 
all weakly-increasing weight vectors. If $\mathcal{F}$ is slope-decreasing, then we are in the same position as above and the maximum realises $\Phi(\mathcal{F})$; so in this case

$$
\Psi\left(\mathcal{F}, W^{0}\right) \leq \Psi\left(\mathcal{F}, W^{\max }\right) \leq \sup \Phi
$$

as desired. If $\mathcal{F}$ is not slope increasing, then the maximum occurs at a vector $W^{\max }$ with $w_{i}^{\max }=w_{i+1}^{\max }$ for some $i$. Then, let $\mathcal{F}^{\prime}$ be the flag obtained from $\mathcal{F}$ by deleting $E_{i}$ and let $W^{\prime}$ be the weight vector for $\mathcal{F}^{\prime}$ obtained from $W^{\max }$ in the obvious way. In the associated sum of quotients, we are replacing the original pair $Q_{i} \oplus Q_{i+1}$ by a bundle $Q_{i}^{\prime}=E_{i+1} / E_{i-1}$. The rank of $Q_{i}^{\prime}$ is the sum $r_{i}+r_{i-1}$ and the degree of $Q_{i}^{\prime}$ is $d_{i}+d_{i-1}$. Thus, $\Psi\left(\mathcal{F}^{\prime}, W^{\prime}\right)=\Psi\left(\mathcal{F}, W^{\text {max }}\right)$. So, we have

$$
\Psi\left(\mathcal{F}, W^{0}\right) \leq \Psi\left(\mathcal{F}, W^{\max }\right)=\Psi\left(\mathcal{F}^{\prime}, W^{\prime}\right) \leq \sup \Phi,
$$

where in the last inequality, we use the inductive hypothesis. This completes the proof.

In sum, the version of the Atiyah-Bott result which is closer to our Theorem is the statement that

$$
\inf _{A}\|F(A)\|=\sup _{\mathcal{F}, W} \Psi(\mathcal{F}, W)
$$

which is completely equivalent to the previous formulation by the elementary Lemma above. One could probably prove one direction here (i.e., that $\inf _{A} \geq \sup _{\mathcal{F}, W}$ ) by using a finite-dimensional approximation argument in the manner of Wang [22], just as we will do for the Calabi functional. However, there would not be much point to this, in view of the simple and direct differential geometric proof.

\section{Differential Geometric asymptotics}

In this section we relate the Calabi functional (and its $L^{q}$ variants) to the norm of a matrix $\underline{M}$ associated to a projective variety. Let $z_{\alpha}$ be standard homogeneous coordinates on $\mathbf{C P}^{N}$ and let $h_{\alpha \beta}$ be the function

$$
h_{\alpha \beta}=\frac{z_{\alpha} \bar{z}_{\beta}}{\|\underline{z}\|^{2}}
$$

on $\mathbf{C P}^{N}$. For a smooth projective variety $V \subset \mathbf{C P}^{N}$ of dimension $n$, we define a self-adjoint matrix $M=M(V)$ with entries

$$
M_{\alpha \beta}=\int_{V} h_{\alpha \beta} d \mu_{F S},
$$

where $d \mu_{F S}$ is the standard volume form induced from the Fubini-Study metric (normalised so that the volume of $V$ is equal to its degree divided by $n$ !). Let $\underline{M}$ be the trace-free part of $M$. Since the sum of the $h_{\alpha \alpha}$ is 1 we have $\underline{M}=M-\frac{\operatorname{Vol}(V)}{N+1} 1$. We recall that for any $q>1$, the $q$-norm 
of a self-adjoint matrix $T$ is defined by

$$
\|T\|_{q}^{q}=\sum\left|\lambda_{\alpha}\right|^{q}
$$

where $\lambda_{\alpha}$ are the eigenvalues, repeated according to multiplicity.

Now, let $(X, L)$ be an abstract polarised variety, as before. For large $k$, the sections of $L^{k}$ define a projective embedding of $X$, i.e., a choice of basis of $H^{0}\left(L^{k}\right)$ yields a specific projective variety $V$.

Proposition 1. Let $\omega_{0}$ be a Kahler metric on $X$ in the class $c_{1}(L)$. Then, for large enough $k$, there is a basis of $H^{0}\left(L^{k}\right)$ yielding a projective embedding $X \rightarrow V_{k} \subset \mathbf{C P}^{N_{k}}$ with for, any $q \geq 1$,

$$
\left\|\underline{M}\left(V_{k}\right)\right\|_{q} \leq k^{(n / q)-1}\left\|S\left(\omega_{0}\right)-\hat{S}\right\|_{L^{q}}+O\left(k^{(n / q)-2}\right) .
$$

The proof is a straightforward application of the asymptotic expansion for the density of states function (see references in Section 1) which we now recall. Let ||$_{0}$ be a Hermitian metric on $L$ whose associated curvature form is $-2 \pi i \omega_{0}$. We write ||$_{0}$ also for the induced metric on $L^{k}$. Then, endow $H^{0}\left(L^{k}\right)$ with the standard $L^{2}$-norm defined by the volume form $d \mu_{0}$ of the fixed metric $\omega_{0}$ and the fibre metric ||$_{0}$ on $L^{k}$. Define a function $\rho_{k}$ on $X$ by

$$
\rho_{k}=\sum\left|s_{\alpha}\right|^{2}
$$

for any orthonormal basis $s_{\alpha}$ of $H^{0}\left(L^{k}\right)$. Then the statement we need is that

$$
\rho_{k}=k^{n}\left(1+k^{-1} \eta_{k}\right)
$$

where the functions $\eta_{k}$ converge (in $C^{\infty}$ ) to $S\left(\omega_{0}\right)$ as $k \rightarrow \infty$; in fact $\eta_{k}=S+O\left(k^{-1}\right)$.

The construction of the projective embedding we need is the most obvious one. For each $k$, we take any orthonormal basis $s_{\alpha}$ of $H^{0}\left(X, L^{k}\right)$ and define a new fibre metric on the line bundle $L$ by

$$
||_{*}^{2}=\frac{1}{\rho_{k}^{1 / k}} \text {. }
$$

Then, denoting the induced metric on $L^{k}$ also by ||$_{*}$,

$$
\sum\left|s_{\alpha}\right|_{*}^{2}=1 \text {. }
$$

The pull-back of the Fubini-Study metric under the embedding is $k \omega$ where

$$
\omega=\omega_{0}+k^{-1} \frac{i}{2 \pi} \bar{\partial} \partial\left(\log \left(1+k^{-1} \eta_{k}\right)\right) .
$$

Write the volume form of $\omega$ as

$$
\left(1+k^{-2} \nu_{k}\right) d \mu_{0} .
$$


It is clear, from the fact that the sequence $\eta_{k}$ is bounded, that the $\nu_{k}$ are bounded. The functions $h_{\alpha \beta}$ pull back to under the projective embedding to $\left(s_{\alpha}, s_{\beta}\right)_{*}$. So, we have

$$
\int_{V_{k}} h_{\alpha \beta} d \mu_{F S}=\int_{X}\left(s_{\alpha}, s_{\beta}\right)_{0}\left(\frac{1+k^{-2} \nu_{k}}{1+k^{-1} \eta_{k}}\right) d \mu_{0} .
$$

We do not change the norm of $\underline{M}\left(V_{k}\right)$ if we apply a unitary transformation to $\mathbf{C}^{N+1}$. In other words, if we make a different choice of orthonormal basis $s_{\alpha}$. Thus, we can choose the basis so that $M$ is a diagonal matrix with diagonal entries $m_{\alpha}$ where

$$
m_{\alpha}=\int_{X}\left|s_{\alpha}\right|_{0}^{2}\left(\frac{1+k^{-2} \nu_{k}}{1+k^{-1} \eta_{k}}\right) d \mu_{0}
$$

Now, the dimension $N+1$ is the integral of $\rho_{k}$ over $X$, and it follows that the trace-free part $\underline{M}$ of $M$ is the diagonal matrix with diagonal entries

$$
\underline{m}_{\alpha}=m_{\alpha}-\frac{1}{1+\hat{\eta}_{k} k^{-1}},
$$

where $\hat{\eta}_{k}$ is the average value of $\eta_{k}$. Then, we obtain $\underline{m}_{\alpha}=k^{-1}\left(b_{\alpha}+\epsilon_{\alpha}\right)$ where

$$
b_{\alpha}=\int_{X}\left|s_{\alpha}\right|_{0}^{2}\left(\hat{\eta_{k}}-\eta_{k}\right) d \mu_{0},
$$

and $\epsilon_{\alpha}$ is $O\left(k^{-1}\right)$. Now write

$$
\left|s_{\alpha}\right|_{0}^{2}\left|\eta_{k}-\hat{\eta_{k}}\right|=\left|s_{\alpha}\right|_{0}^{2 / p}\left|s_{\alpha}\right|_{0}^{2 / q}\left|\eta_{k}-\hat{\eta_{k}}\right|,
$$

where $p$ is the index conjugate to $q$. Apply Holder's inequality to get

$$
\left|b_{\alpha}\right| \leq\left(\int_{X}\left|s_{\alpha}\right|_{0}^{2} d \mu_{0}\right)^{1 / p}\left(\int_{X}\left|s_{\alpha}\right|_{0}^{2}\left|\eta_{k}-\hat{\eta_{k}}\right|^{q} d \mu_{0}\right)^{1 / q} .
$$

But since the $s_{\alpha}$ are orthonormal, this gives

$$
\left|b_{\alpha}\right|^{q} \leq \int_{X}\left|s_{\alpha}\right|_{0}^{2}\left|\eta_{k}-\hat{\eta_{k}}\right|^{q} d \mu_{0} .
$$

Summing over $\alpha$ and using the asymptotic statement again in the weak form

we obtain

$$
\sum\left|s_{\alpha}\right|_{0}^{2}=k^{n}+O\left(k^{n-1}\right)
$$

$$
\sum_{\alpha}\left|b_{\alpha}\right|^{q} \leq\left(k^{n}+C k^{n-1}\right) \int_{X}\left|\eta_{k}-\hat{\eta_{k}}\right|^{q} d \mu_{0}
$$

Now,

$$
\|\underline{M}\|_{q} \leq k^{-1}\left(\|B\|_{q}+\|E\|_{q}\right)
$$

where $B, E$ are the diagonal matrices with entries $b_{\alpha}, \epsilon_{\alpha}$ respectively. We have

$$
\|B\|_{q} \leq k^{n / q}\left(1+C k^{-1}\right)^{1 / q}\left\|\eta_{k}-\hat{\eta_{k}}\right\|_{L^{q}} \leq k^{n / q}\left(1+\frac{C}{q} k^{-1}\right)\left\|\eta_{k}-\hat{\eta_{k}}\right\|_{L^{q}},
$$


and $\|E\|=O\left(k^{(n / q)-1}\right)$ since the dimension $N+1$ is $O\left(k^{n}\right)$. This gives

$$
\|\underline{M}\|_{q} \leq k^{(n / q)-1}\left\|\eta_{k}-\hat{\eta_{k}}\right\|_{L^{q}}+O\left(k^{(n / q)-2}\right),
$$

and our result follows from the fact that $\eta_{k}-\hat{\eta_{k}}=\left(S\left(\omega_{0}\right)-\hat{S}\right)+O\left(k^{-1}\right)$.

\section{The finite-dimensional argument}

Consider a $\mathbf{C}^{*}$ action on $\mathbf{C P}^{N}$ induced by a 1-parameter subgroup $\rho: \mathbf{C}^{*} \rightarrow G L(N+1)$. Let $V$ be a smooth $n$ dimensional projective variety and set $V^{t}=\rho(t)(V)$. Then, it follows from standard theory that the $V^{t}$ converge as $t \rightarrow 0$ to some algebraic cycle $V^{0}$. Thus, $V^{0}$ is a formal sum

$$
V^{0}=\sum m_{i} W_{i}
$$

where the mulptiplicities $m_{i}$ are positive integers and $W_{i}$ are irreducible $n$-dimensional projective varieties. This convergence can be understood at various levels, but the crucial point for us is that the $V^{t}$ converge to $V^{0}$ in the sense of currents. So for any smooth test form $\phi$

$$
\int_{V^{t}} \phi \rightarrow \int_{V^{0}} \phi=\sum m_{i} \int_{W_{i}} \phi
$$

Now, suppose that $\rho$ maps $S^{1} \subset \mathbf{C}^{*}$ to the unitary group. Thus, the infinitesimal generator $A$ of $\rho$ is a Hermitian matrix; as usual, we write $\underline{A}$ for the trace-free part of $A$. Let $h$ be the real valued function

$$
h=\sum_{\alpha \beta} A_{\alpha \beta} h_{\alpha \beta}
$$

on $\mathbf{C P}^{N}$. This is a Hamiltonian for the action of $S^{1}$ on the projective space. Let

$$
I\left(A, V^{0}\right)=\int_{V(0)} h d \mu_{F S}=\frac{1}{n !} \int_{V(0)} h \omega_{F S}^{n},
$$

where $\omega_{F S}$ is the Fubini-Study form, and set

$$
F C h\left(A, V^{0}\right)=\frac{\operatorname{Vol}\left(V^{0}\right)}{N+1} \operatorname{Tr}(A)-I\left(A, V^{0}\right),
$$

where the volume of $V^{0}$ has the obvious meaning.

Proposition 2. Suppose that $F C h\left(A, V^{0}\right)<0$. Then for any conjugate indices $p, q$, we have

$$
\|\underline{M}(V)\|_{q} \geq \frac{-F C h(A, V(0))}{\|\underline{A}\|_{p}} .
$$

To prove this, we consider the function

$$
f(t)=\operatorname{Tr}\left(\underline{A} \underline{M}\left(V^{t}\right)\right),
$$

for $t \in \mathbf{R}^{*}$. The crucial point is that this is increasing with $t$. From one point of view, this follows from the fact that $\underline{M}$ is a moment map, 
and from the general theory of such maps, see Section 6.5.2 in [11], for example. If $\mu$ is a moment map for an isometric action of a group $G$ on a Kahler manifold, we have, for any $a \in \operatorname{Lie}(G)$,

$$
\frac{d}{d s}\langle\mu(\exp (s a) X), a\rangle=\left|\frac{d}{d s} \exp (s a) X\right|^{2} \geq 0 .
$$

Then, our assertion follows (taking $t=e^{s}$ ) from the fact established in [9] that $\underline{M}$ is a moment map for the $S U(N+1)$ action on the set of varieties projectively equivalent to $V$, with a suitable Kahler structure. An equivalent fact was used in the earlier work of Zhang [25], and the relation between this and the constructions of [9] is explained in [15], [16]. This monotonicity property is often stated in the literature as the convexity of a certain function, and other direct proofs are given in [10], Prop. 1, and [16], Lemma 3.1, so we will not discuss the matter further here. Given this monotonicity property, we argue as follows. We have

$$
\operatorname{Tr}\left(A M\left(V^{t}\right)\right)=\int_{V\left(e^{t}\right)} h_{A} d \mu_{F S}
$$

and $\underline{M}=M-\frac{\operatorname{Vol}(V)}{N+1} 1$. So

$$
f(t)=\operatorname{Tr}\left(\underline{A} \underline{M}\left(V^{t}\right)\right)=\operatorname{Tr}\left(A \underline{M}\left(V^{t}\right)\right)=\int_{V^{t}} h_{A} d \mu_{F S}-\frac{\operatorname{Vol}}{N+1} \operatorname{Tr} A .
$$

Hence, the limit of $f(t)$ as $t \rightarrow 0$ is $-F C h\left(A, V^{0}\right)$, which is positive by hypothesis. Since the function $f$ is increasing

$$
|f(t)|=f(t) \geq-F C h\left(A, V^{0}\right)
$$

for all $t$. In particular, taking $t=1$, we have

$$
\operatorname{Tr}(\underline{A} \underline{M}(V)) \geq-F C h\left(A, V^{0}\right) .
$$

Now, use the fact that for, any Hermitian matrices $S, T$

$$
|\operatorname{Tr}(S T)| \leq\|S\|_{p}\|T\|_{q},
$$

to obtain the required result.

We remark that all of this dicussion can be placed in the context of the action of $G L(N+1)$ on the space of Chow vectors, as explained in [15], [16], and the criterion $F C h\left(A, V^{0}\right)<0$ is the standard HilbertMumford criterion for a destabilising 1-parameter subgroup. On the other hand, the criterion can also be placed in a dynamical, Riemannian geometry context. Let $P$ be a compact Riemannian manifold, $S \subset P$ a submanifold and let $h$ be a real-valued function on $P$. Let $\phi_{s}$ be the flow generated by the ( $h$-decreasing) gradient of $h$ and suppose that the $\phi_{s}(S)$ converge to some varifold $S^{\prime}$ as $s \rightarrow \infty$. Say that $S$ has property $\left(^{*}\right)$ with respect to $h$ if the mean value of $h$ over $S^{\prime}$ is less than the mean 
value of $h$ on $P$. Then, a variety $V \subset \mathbf{C P}^{N}$ is Chow stable if it has property $(*)$ with respect to all the functions $h_{A}$. This is because

$$
-F C h\left(A, V^{0}\right)=\operatorname{Vol}\left(V^{0}\right)\left(\frac{I}{\operatorname{Vol}\left(V^{0}\right)}-\frac{\operatorname{Tr}(A)}{N+1}\right),
$$

and $\operatorname{Tr}(A) / N+1$ is the mean value of $h_{A}$ on $\mathbf{C} \mathbf{P}^{N}$.

\section{Algebro-geometric asymptotics}

We now go back to a test configuration $\mathcal{X}$ for $(X, L)$. We can suppose that the parameter $r$ is 1 . The essential point is that for large enough $k$, the configuration can be realised by a $\mathbf{C}^{*}$-action on an ambient projective space. That is to say, $\mathcal{X}$ can be embedded as a $\mathbf{C}^{*}$ invariant subscheme in the product $\mathbf{P}\left(U_{k}^{*}\right) \times \mathbf{C}$, extending the embedding of the central fibre $X_{0} \subset \mathcal{X}$ by the complete linear system $U_{k}$ in $\mathbf{P}\left(U_{k}^{*}\right)=\mathbf{P}\left(U_{k}^{*}\right) \times\{0\}$. This is explained in [17]. In essence, we consider the $\mathbf{C}^{*}$-equivariant bundle $\mathcal{U}=\pi_{*} \mathcal{L}^{k}$ over $\mathbf{C}$ and pick an equivariant trivialisation $\mathcal{U} \cong \mathbf{C} \times U_{k}$. There is no loss of generality in supposing that $\mathcal{L}^{k}$ is very ample on all fibres for all $k \geq 1$; then we obtain the embedding of $\mathcal{X}$ from the fibrewise embeddings of $X_{t}$ in $\mathbf{P}\left(\mathcal{U}_{t}^{*}\right)$ under this trivialisation. Let $\left|X_{0}\right|$ denote the cycle in $\mathbf{P}\left(U_{k}^{*}\right)$ associated to the scheme $X_{0}$. Then, we are precisely in the situation considered above with a 1-parameter subgroup acting on $\mathbf{P}^{N+1}$, generated by $A_{k}$. If we write $V^{t}$ for the image of $X_{t} \times\{t\} \subset \mathcal{X} \subset \mathbf{P} \times \mathbf{C}$ under the projection map to $\mathbf{P}$, then $V^{t}=\rho(t)\left(V_{1}\right)$ and the $V^{t}$ converge to the cycle $\left|X_{0}\right|$ as $t \rightarrow 0$.

We need a general fact about the choice of equivariant trivialisation of the bundle $\mathcal{U}$.

Lemma 2. Let $E$ be $\mathbf{C}^{*}$-equivariant bundle over $\mathbf{C}$ and let $H$ be a Hermitian metric on the fibre $E_{1}$. Then, there is an equivariant trivialisation $E \cong \mathbf{C} \times E_{0}$ which takes $H$ to a Hermitian metric on the central fibre $E_{0}$ which is preserved by the action of $S^{1} \subset \mathbf{C}^{*}$ on $E_{0}$.

To see this, consider the weight spaces $E_{0}=\bigoplus V_{i}$ say, where $\mathbf{C}^{*}$ acts with weight $w_{i}$ on $V_{i}$ and the ordering is chosen so that $w_{1}<w_{2}<\ldots$. Let $\mathcal{F}$ be the flag

$$
V_{1} \subset V_{1} \oplus V_{2} \subset V_{1} \oplus V_{2} \oplus V_{3} \ldots,
$$

in $E_{0}$ and let $\alpha: E_{0} \rightarrow E_{0}$ be a linear map which preserves the flag $\mathcal{F}$. Thus, $\alpha$ has a block matrix description $\alpha_{i j}: V_{i} \rightarrow V_{j}$ with $\alpha_{i j}=0$ for $i>$ $j$. For any $t \in \mathbf{C}$, we define $\alpha(t)$ with blocks $t^{w_{j}-w_{i}} \alpha_{i j}$. This defines a $\mathbf{C}^{*}$ equivariant automorphism of the trivial bundle $\mathbf{C} \times E_{0}$ equal to $\alpha$ on the fibre $\{1\} \times E_{0}$. Conversely, all equivariant automorphisms arise in this way. What this means is that in the fibre $E_{1}$ of our equivariant bundle $E$, there is a canonical flag and a choice of equivariant trivialisation is 
equivalent to a choice of compatible direct sum splitting of $E_{1}$. Thus, the proof of the Lemma is simply to take the direct sum splitting furnished by the succesive orthogonal complements in the flag in $E_{1}$ using the metric $H$, and then take the corresponding equivariant trivialisation.

Applying the Lemma to the bundle $\mathcal{U}$, we see that, given any metric on $H^{0}\left(X, L^{k}\right)$, we may choose our representation of $\mathcal{X}$ so that the 1 parameter subgroup takes the circle to unitary transformations of $U_{k}$, with respect to the metric arising from the identification of $U_{k}$ with $H^{0}\left(X, L^{k}\right)$. (Of course, we can choose a basis so that the given metric is identified with the standard one on $\mathbf{C}^{N+1}$.) Then, we have a numerical invariant

$$
I_{k}=I\left(A_{k},\left|X_{0}\right|\right)=\int_{\left|X_{0}\right|} h d \mu_{F S}
$$

as above.

Proposition 3. The integral $I_{k}$ is equal to the leading term $b_{1} k^{n+1}$ in the Hilbert polynomial for $\operatorname{Tr}\left(A_{k}\right)$.

Assuming this for the moment, we go on to complete the proof of our main result. Observe that it is equivalent to consider a fixed embedding $X \rightarrow V=V^{1}$ with a variable metric on the underlying vector space $\mathbf{C}^{N+1}$ (as we are doing here) or to consider a fixed metric on $\mathbf{C}^{N+1}$ and varying the embedding by projective transformations (as we considered in Section 3). Set

$$
F C h_{k}=F C h\left(A_{k},\left|X_{0}\right|\right)=\frac{\operatorname{Vol}(X, k \omega)}{\operatorname{dim} U_{k}} \operatorname{Tr} A_{k}-I_{k},
$$

as in the previous section. Thus,

$$
F C h_{k}=\left(\frac{a_{0} k^{n}}{a_{0} k^{n}+a_{1} k^{n-1}+\cdots}\right)\left(b_{0} k^{n+1}+b_{1} k^{n}+\cdots\right)-b_{0} k^{n+1} .
$$

So

$$
F C h_{k}=\left(b_{1}-\frac{a_{1} b_{0}}{a_{0}}\right) k^{n}+O\left(k^{n-1}\right)=F_{\mathcal{X}} k^{n}+O\left(k^{n-1}\right) .
$$

Now, Theorem 2 is vacuous if $F_{\mathcal{X}} \geq 0$, so suppose that $F_{\mathcal{X}}<0$. Then, (15) means that $F C h_{k}<0$ for large $k$. Thus, we can apply Proposition 2 to deduce that for the embedding $X \rightarrow V=V(1)$

$$
\|\underline{M}(V)\|_{q} \geq-\frac{F C h_{k}}{\left\|\underline{A}_{k}\right\|_{p}} .
$$

If $p$ is an even integer, then $\left\|A_{k}\right\|_{p}^{p}=\operatorname{Tr} \underline{A}^{p}$, so

$$
\frac{F C h_{k}}{\left\|\underline{A}_{k}\right\|_{p}}=k^{(n / q)-1} \frac{F \mathcal{X}}{N_{p}}+O\left(k^{n / q)-2}\right) .
$$


Thus

$$
\|\underline{M}(V)\|_{q} \geq-k^{(n / q)-1} \frac{F_{\mathcal{X}}}{N_{p}}+O\left(k^{(n / q)-2}\right) .
$$

Then, Theorem 2 follows from this lower bound combined with Proposition 1 , since if there was a Kahler metric $\omega_{0}$ with $\|S-\hat{S}\|_{L^{q}}<$ $\hat{\Psi}_{p}(\mathcal{X})$, we would get, for large $k$, an embedding $V$ with $\|\underline{M}(V)\|_{q}<$ $-k^{(n / q)-1} F_{\mathcal{X}} / N_{p}$ in contradiction to (16).

5.1. Proof of Proposition 3. First observe that if we have proved that $\operatorname{Tr}\left(A_{k}\right) \sim k^{n+1} I_{1}$, then replacing $L$ by $L^{s}$ it will follow that $\operatorname{Tr}\left(A_{s k}\right)$ $\sim(s k)^{n+1} I_{s}$ and so that $I_{s}=s^{n+1} I_{1}$. (This can also be seen directly. If we assume that the powers of sections in $H^{0}\left(X_{0}, \mathcal{L}\right)$ generate $H^{0}\left(X_{0}, \mathcal{L}^{s}\right)$ then a choice of metric on the first space yields a natural metric on the second. With these metrics, the integrand defining $I_{s}$ is $s^{n+1}$ times that defining $I_{1}$, pointwise on $|X|$.) To sum up, our task is to establish the asymptotic relation

$$
\operatorname{Tr}\left(A_{k}\right) \sim k^{n+1} I
$$

where we recall that

- $X_{0}$ is a $\mathbf{C}^{*}$-equivariant subscheme of $\mathbf{C P}^{N}$ for the action generated by $A=A_{1}$ on $\mathbf{C}^{N+1}=H^{0}\left(X_{0}, \mathcal{O}(1)\right)$;

- $A_{k}$ is the generator of the induced action on $H^{0}\left(X_{0}, \mathcal{O}(k)\right)$;

- We fix an $S^{1}$-invariant metric on $\mathbf{C}^{N+1}$. Then, $I$ is the integral of $h \omega_{F S}^{n} / n$ ! over the cycle $\left|X_{0}\right|$ associated to $X_{0}$, where $\omega_{F S}$ is the Fubini-Study metric on $\mathbf{C P}^{N}$ and $h$ is the function on $\mathbf{C P}^{N}$ associated to $A$.

If $X_{0}$ is smooth, the relation (17) is rather standard. The key point is that the function $h$ is a Hamiltonian for the $S^{1}$ action on $\mathbf{C P}^{N}$. The desired result is then obtained from the equivariant Riemann-Roch Theorem and the de Rham model for equivariant cohmology, as explained by Atiyah and Bott in [2]; see the discussion in Section 2 of [8]. Our problem is to extend this discussion to the case when $X_{0}$ is a singular variety or scheme. What we do take as known is the corresponding nonequivariant result. That is, if $Z \subset \mathbf{C P}^{M}$ is an $m$-dimensional projective scheme, then

$$
\operatorname{dim} H^{0}(Z, \mathcal{O}(k)) \sim \frac{D}{m !} k^{m},
$$

where $D$ is the degree of $Z$, which is the integral over the cycle $|Z|$ of $\omega_{F S}^{m}$. (The proof of this can be reduced to the case $m=0$ by taking hyperplane sections. Thus, the assertion is essentially that the notions of multiplicity defined algebraically or by currents agree.) We will explain how to prove the equivariant result by reducing to (18).

We consider the following general situation. Let $P \rightarrow B, Q \rightarrow F$ be a pair of principle $S^{1}$-bundles over manifolds $B, F$. So, we have $S^{1}$ actions 
$\sigma_{P}, \sigma_{Q}$ say and vector fields $v_{P}, v_{Q}$ on $P, Q$. Suppose, in addition, that $Q \rightarrow F$ is an $S^{1}$-equivariant circle bundle, so we have another action $\rho$ on $Q$, commuting with $\sigma_{Q}$, and another vector field, $w$ say, on $Q$. We also denote the induced action on $F$ by $\rho$. Now, we can form the associated bundle

$$
M=P \times_{\sigma_{P}, \rho} F .
$$

Thus, $M$ is a bundle over $B$ with fibre $F$. We can also form

$$
\Pi=P \times_{\sigma_{P}, \rho} Q .
$$

Then, $\Pi$ is a circle bundle over $M$. Suppose now that we have connections on the bundles $P \rightarrow B, Q \rightarrow F$ and that the connection on $Q$ is preserved by $\rho$. Thus, we have 1 -forms $\alpha_{P}, \alpha_{Q}$ on $P, Q$ and

$$
\alpha_{P}\left(v_{P}\right)=1, \alpha_{Q}\left(v_{Q}\right)=1, \alpha_{Q}(w)=-H,
$$

where $H$ is an $S^{1} \times S^{1}$-invariant function on $Q$, which can also be regarded as a function on $F$ or on $M$. Now, the connection on $P$ defines a splitting of the tangent bundle of the associated bundle $M$, which we express rather loosely as

$$
T M=T F \oplus T B .
$$

Via this decomposition, the curvature forms $\omega_{P}, \omega_{Q}$ can naturally be regarded as 2 -forms on $M$. The key point is that there is a connection on $\Pi \rightarrow M$ with curvature

$$
\Omega=\omega_{Q}+H \omega_{P} .
$$

To see this, we pull everything back to $P \times Q$ where we consider the 1 -form

$$
\beta=\alpha_{Q}+H \alpha_{P}
$$

(making an obvious simplification in notation). Then, $\beta$ vanishes on the generator $v_{P}+w$ of the action $\left(\sigma_{P}, \rho\right)$ and so descends to a form $\beta$ on $\Pi=P \times_{\sigma_{P}, \rho} Q$. Since $\beta\left(v_{Q}\right)=1$ the equivariant 1 -form $\beta$ furnishes a connection on $\Pi$. We have (again making various abuses of notation)

$$
d \beta=\omega_{Q}+H \omega_{P}+d H \wedge \alpha_{P} .
$$

This gives the lift of the curvature form of the connection on $\Pi$ to $P \times Q$. The definition of the horizontal subspace defining the splitting $T M=T F \oplus T B$ means that $\alpha_{P}$ vanishes on the vectors representing $T M$, so we see that the connection $\beta$ has curvature $\Omega$.

Now, suppose that $B$ is a compact oriented $2 r$-manifold and let $S$ be an $S^{1}$-invariant oriented $2 n$-dimensional submanifold of $F$, not necessarily closed. This defines a corresponding submanifold $\tilde{S}$ in $M$ (so $\tilde{S}$ is a bundle over $B$ with fibre $S$ ). Then, it is clear, by integrating over the fibres, that

$$
\frac{1}{(n+r) !} \int_{\tilde{S}} \Omega^{n+r}=\frac{1}{r !} \int_{B} \omega_{P}^{r} \frac{1}{n !} \int_{S} H^{n} \omega_{Q}^{n}
$$


We apply this to the case when $B$ is the Riemann sphere $\mathbf{C P}^{1}$ and $F$ is $\mathbf{C P}^{N}$ with the bundles $P, Q$ being the Hopf fibrations. We take the action $\rho$ to be that induced by the given action on $\mathbf{C}^{n+1}$. Then, the function $H$ considered above becomes the function $h$ by the standard discussion of Hamiltonians and equivariant classes, as in [2]. We then have a manifold $M$, which is just the projectivization of a vector bundle over $\mathbf{C P}^{1}$, and a circle bundle $\Pi \rightarrow M$ which clearly corresponds to a holomorphic line bundle say $\mathcal{V} \rightarrow M$. Notice that, going back to our original data, we could change the generator $A$ to $A+\nu 1$ for any integer $\nu$. This changes the function $h$ to $h+\nu$ and, by applying (18), does not affect the truth of the result we seek. So, we can suppose that $h$ is positive and this means that $\mathcal{V}$ is a positive line bundle over $M$. Thus, we can embed $M$ as a projective variety in some $\mathbf{C P}^{\mu}$, with $\mathcal{V}^{s}=\mathcal{O}(1)$, and there will be no loss in generality in supposing that $s=1$.

Now, consider our $\mathbf{C}^{*}$-invariant scheme $X_{0} \subset \mathbf{C} \mathbf{P}^{N}$. Clearly, we get a corresponding scheme $Z$ inside $M$, fibering as $\pi: Z \rightarrow \mathbf{C P}^{1}$ with fibre $X_{0}$. For any $k$, we can identify $H^{0}\left(Z ; \mathcal{V}^{k}\right)$ with the sections of the vector bundle $\pi_{*}\left(\mathcal{V}^{k}\right)$ over $\mathbf{C P}^{1}$. Now, take the eigenspace decomposition

$$
H^{0}\left(X_{0}, \mathcal{O}(k)\right)=\bigoplus V_{i}
$$

where $A_{k}$ acts as $w_{i}$ on $U_{i}$. Then

$$
\pi_{*}\left(\mathcal{V}^{k}\right)=\bigoplus U_{i} \otimes \mathcal{O}\left(w_{i}\right)
$$

It follows that

$$
\operatorname{dim} H^{0}\left(Z, \mathcal{V}^{k}\right)=\sum \operatorname{dim} U_{i}\left(w_{i}+1\right)=\operatorname{Tr} A_{k}+\operatorname{dim} H^{0}\left(X_{0}, \mathcal{O}(k)\right) .
$$

We apply (18) to the projective scheme $Z \subset M \subset \mathbf{C P}^{M}$. This shows that

$$
\operatorname{Tr} A_{k} \sim D k^{n+1}
$$

where $D$ is the degree of $Z$. This degree is given by integrating $\Omega^{n+1}$ over the cycle $|Z|$, for any smooth form $\Omega$ on $M$ representing $c_{1}(\mathcal{V})$. Taking the form given by our construction above, taking $S$ to be the smooth points in $|Z|$ and taking due account of multiplicity, we see that the degree is given by the integral $I$, and our result follows.

Note that the same argument (taking $B$ to be $\mathbf{C P}^{r}$ ) shows that for any positive integer $r$

$$
\operatorname{Tr} A_{k}^{r} \sim k^{n+r} \int_{\left|X_{0}\right|} h^{r} d \mu_{F S}, \quad \operatorname{Tr} \underline{A}_{k}^{r} \sim k^{n+r} \int_{\left|X_{0}\right|}(h-\hat{h})^{r} d \mu_{F S} .
$$

Thus, the invariant $N_{p}$ is the $L^{p}$ norm of $h-\hat{h}$ on $\left|X_{0}\right|$. One consequence of this is that we can extend Theorem 2 to the case $q=1, p=\infty$, defining $N_{\infty}(\mathcal{X})=\|h-\hat{h}\|_{L^{\infty}\left(\left|X_{0}\right|\right)}$. It would be interesting to extend Theorem 2 to general real exponents $p, q$ with

$$
N_{p}=\|h-\hat{h}\|_{L^{p}\left(\left|X_{0}\right|\right)} .
$$




\section{References}

[1] M.F. Atiyah \& R. Bott, The Yang-Mills equations over Riemann surfaces, Phil. Trans. Roy. Soc. London A 308 (1983) 523-615, MR 0702806, Zbl 0509.14014.

[2] _ The moment map and equivariant cohomology, Topology 23 (1984) 128, MR 0721448, Zbl 0521.58025.

[3] E. Calabi, Extremal Kahler metrics, in 'Seminar on differential geometry' (S.T. Yau, ed.), 259-290, Annals of Math. Studies, 102, Princeton U.P., 1982, MR 0645743, Zbl 0487.53057.

[4] D. Catlin, The Bergman kernel and a theorem of Tian, in 'Analysis and geometry in several complex variables' (Katata, 1997), 1-23, Birkhauser, Boston, 1999, MR 1699887, Zbl 0941.32002.

[5] X.-X. Chen, The space of Kahler metrics, J. Differential Geometry 56 (2000) 189-234, MR 1863016, Zbl 1041.58003.

[6] X.-X. Chen \& G. Tian, Partial regularity for homogeneous complex MongeAmpère equation, C.R. Math. Acad. Sci. Paris 340(5) (2005) 337-340, MR 2127106.

[7] S.K. Donaldson, Remarks on gauge theory, complex geometry and four-manifold topology, in 'Fields Medallists' Lectures' (Atiyah, Iagolnitze, eds.), World Scientific, 1997, MR 1622931.

[8] Scalar curvature and stability of toric varieties, J. Differential Geometry 62 (2002) 289-349, MR 1988506.

[9] Scalar curvature and projective embeddings, I, J. Differential Geometry 59 (2001) 479-522, MR 1916953, Zbl 1052.32017.

[10] Scalar curvature and projective embeddings, II, Quarterly Jour. Math. 56(3) (2005) 345-356, MR 2161248.

[11] S. Donaldson \& P. Kronheimer, The geometry of four-manifolds, Oxford U.P., 1990, MR 1079726, Zbl 0820.57002.

[12] F.C. Kirwan, Cohomology of quotients in symplectic and algebraic geometry, Princeton U.P., 1984, MR 0766741, Zbl 0553.14020.

[13] Z. Lu, On the lower order terms of the asymptotic expansion of Tian-YauZelditch, Amer. J. Math. 122 (2000) 235-273, MR 1749048, Zbl 0972.53042.

[14] T. Mabuchi, An energy-theoretic approach to the Hitchin-Kobayashi correspondence for manifolds, I, Invent. Math. 159(2) (2005) 225-243, MR 2116275.

[15] D. Phong \& J. Sturm, Scalar curvature, moment maps and the Deligne pairing, Amer. J. Math. 126 (2004) 693-712, MR 2058389.

[16] Stability, energy functionals and Kahler-Einstein metrics, Commun. Anal. Geom. 11(3) (2003) 565-597, MR 2015757.

[17] J. Ross \& R. Thomas, A study of the Hilbert-Mumford criterion for the stability of projective varieties, preprint AG/0412519.

[18] W. Ruan, Canonical coordinates and Bergman metrics, Commun. Analysis and Geometry 6 (1998) 589-631, MR 1638878, Zbl 0917.53026.

[19] G. Szekelyhidi, Extremal metrics and K-stability, preprint AG/0410401.

[20] G. Tian, On a set of polarized Kahler metrics on algebraic manifolds, J. Differential Geometry 32 (1990) 99-130, MR 1064867, Zbl 0706.53036.

[21] Kahler-Einstein metrics with positive scalar curvature, Inv. Math. 130 (1997) 1-37, MR 1471884, Zbl 0892.53027. 
[22] X. Wang, Balance point and stability of vector bundles over a projective variety, Math. Res. Letters 9 (2002) 393-411, MR 1909652, Zbl 1011.32016.

[23] S.-T. Yau, Open problems in geometry, Enseign. Math. 33 (1987) 109-158.

[24] S. Zelditch, Szegö kernel and a theorem of Tian, Int. Math. Res. Notices 6 (1998) 317-331.

[25] S. Zhang, Heights and reductions of semi-stable varieties, Compositio Math. 104 (1996) 77-105, MR 1420712, Zbl 0924.11055.

IMPERIAL COLLEGE UNIVERSITY OF LONDON DEPARTMENT OF MATHEMATICS 180 Queens Gate, London, SW7 2AZ ENGLAND

E-mail address: s.donaldson@imperial.ac.uk 